\title{
Enseñanza y aprendizaje en resolución de problemas: productos notables
}

\section{Teaching and learning in problem solving: algebraic identity}

Recepción: 7 de Febrero de 2016.

Aceptación 12 de Octubre de 2016.

Alfonso Jiménez Espinosa* Laura Emily Parra Quemba **

Artículo de Reflexión derivado de Investigación

\section{Resumen}

Este artículo presenta resultados parciales de una investigación que tuvo como objetivo: establecer las características de las prácticas de enseñanza y aprendizaje en la resolución de problemas de factorización para el grado octavo, y caracterizar algunos aspectos de las prácticas de enseñanza y aprendizaje de resolución de problemas sobre productos notables. A partir del análisis de tres textos escolares y un cuestionario aplicado a 191 estudiantes, se hace la configuración epistémica y cognitiva del Enfoque Ontosemiótico. Los componentes de idoneidad epistémica, cognitiva y afectiva del enfoque dan relevancia al grado de representatividad del significado institucional, a la apropiación de los conocimientos y al uso de medios tecnológicos. La metodología fue de carácter mixto, y se usó el método de análisis de contenido. Entre los hallazgos se infiere que prevalece el modelo pedagógico tradicional, debido a que las clases de álgebra se fundamentan en las explicaciones de los docentes.

Palabras clave: álgebra, productos notables, enfoque ontosemiótico, resolución de problemas, aprendizaje.

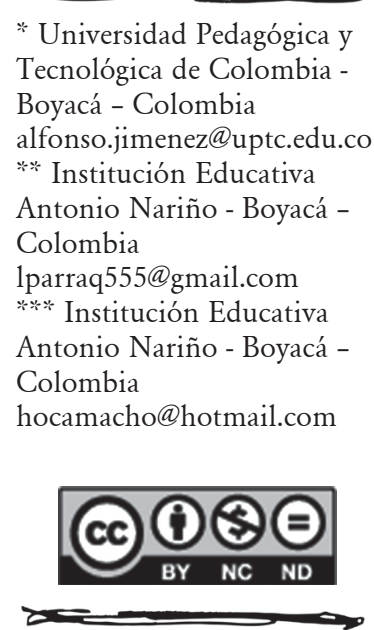

* Universidad Pedagógica y Tecnológica de Colombia Boyacá - Colombia alfonso.j1menez@uptc.edu.co Antonio Nariño - Boyacá Colombia

1parraq555@gmail.com $* * * *$ Institución Educativa Antonio Nariño - Boyacá Colombia

hocamacho@hotmail.com 


\section{Abstract}

This article presents partial results of a research aimed to: establish the characteristics of the practices of teaching and learning in problem solving of algebraic factorization for the eighth grade and characterize some aspects of teaching practices and learning problem solving on algebraic identities. From the analysis of three school texts and a questionnaire applied to 191 students, the epistemic and cognitive configuration of the Ontosemiotic Approach is made. The components of epistemic, cognitive and affective suitability of the approach give relevance to the degree of representativeness of the institutional meaning, to the appropriation of knowledge and to the use of technological means. The methodology was of a mixed nature, and the content analysis method was used. Among the findings it is inferred that the traditional pedagogical model prevails, because the algebra classes are based on the explanations of the teachers.

Keywords: algebra, algebraic identities, ontosemiotic approach, problem solving, learning. 


\section{Introducción}

La educación en Colombia ha pasado por cambios que han generado rupturas en el mejoramiento de la calidad de la educación; las instituciones educativas han tenido que adaptarse e incluir ajustes, entre los que se encuentran atender la cobertura, mejorar la evaluación y los resultados de pruebas, tanto internas como externas, ya que estas reflejan bajos desempeños de los estudiantes. En el aula de clase se enmarcan diferencias en el entorno $\mathrm{y}$ en las particularidades propias de cada lugar, por lo que no es posible solucionar una problemática de igual manera en uno u otro sitio. El maestro es quien vivencia estas singularidades a través de las prácticas y debe ser capaz de enriquecer, transformar o adaptar estos ambientes para optimizar la eficacia de los procesos y obtener más y mejores aprendizajes.

Las prácticas de enseñanza son la puesta en escena y en contexto de la formación técnica y profesional que reciben los maestros en las escuelas normales $\mathrm{y}$ las universidades, y se relacionan directamente con los resultados que se obtienen en el ejercicio pedagógico del maestro con los estudiantes, desde las pruebas externas. Es indudable que la reflexión del maestro es importante para reorganizar el aula de clase a partir del contexto, transformar sus prácticas, organizar y orientar las actividades, limitar el tiempo, continuar con su formación, mejorar las interacciones en que participan los actores de la educación e incorporar innovaciones que se requieren para despertar el interés de los estudiantes.
Las prácticas requieren que el docente se involucre en la investigación, dado que tendrá una relación de aprendizaje tanto de investigador como de investigado, puesto que tiene la oportunidad de observarse desde su ejercicio educativo. En la relación que existe entre el docente y el investigador, son relevantes las experiencias que se vivan en el aula, la autocrítica del docente y la investigación in situ. Clandinin (citado en Sancho \& Martínez, 2014) señala cambios o transiciones fundamentales que constituyen desafíos para la investigación, en particular en el ámbito de la educación. El primero está relacionado con una profunda revisión de la relación entre el investigador y los investigados. Otra es el giro narrativo, los objetos/sujetos de investigación se convierten en individuos biográficos con capacidad de acción y activos constructores de conocimiento y visiones sobre el mundo.

En un estudio realizado sobre la enseñanza, Smith (citado en Nieto, 2002 , p. 78) afirma que existe una pluralidad de acepciones de este vocablo, e intenta sintetizarlas en las siguientes dimensiones: sentido originado y mostrativo, enseñanza como logro, actividad intencional, actividad normativa, actividad interactiva $y$ actividad reflexiva. El sentido originario y mostrativo expone la importancia de la palabra enseñanza y la concibe más allá del entorno educativo. La enseñanza como logro o adquisición de aprendizajes, es planteada para analizarla desde el estudiante. Además, para que adquiera sentido y gran significación, debe producirse aprendizaje.
Las prácticas de enseñanza son la puesta en escena y en contexto de la formación técnica y profesional que reciben los maestros en las escuelas normales y las universidades, y se relacionan directamente con los resultados que se obtienen en el ejercicio pedagógico del maestro con los estudiantes, desde las pruebas externas. 
En las instituciones educativas generalmente se percibe un ambiente de negligencia y desmotivación entre los estudiantes, posiblemente por lo aburridas que les parecen las clases, o la falta de interés tanto del maestro como del estudiante en los procesos de enseñanza y aprendizaje de las matemáticas.
Como actividad intencional y anticipatoria, implica la importancia de la tenacidad que debe imprimir el maestro en su labor y, el reconocimiento de que el éxito de su labor no es total, por la imposibilidad de controlar todas las variables que intervienen en el proceso de aprendizaje, como la motivación y la atención; es relevante que el maestro atienda, analice y comprenda lo que sucede al interior del aula, que realice diagnósticos y cambie su comportamiento para perfeccionar estos procesos y mejorar los resultados. La labor docente está estrechamente relacionada con ciertas condiciones éticas, ya que los valores se dan a través de los programas y objetivos establecidos institucionalmente.

En la enseñanza, como actividad interactiva, el ejercicio profesional ya no consiste solamente en transmitir conocimientos, actitudes y valores sino en dirigir los aprendizajes a través de situaciones problematizantes, dialógicas y de reflexión crítica, en contextos de interacción social, para que los estudiantes aprendan a razonar y a construir soluciones. En cuanto a la enseñanza como actividad reflexiva, es indispensable que el maestro reflexione sobre su propia práctica y, a la vez, sea innovador, para ofrecer cambios drásticos en el aula (Smith citado en Nieto, 2002).

Durante el desarrollo de las clases, los docentes inicialmente orientan el trabajo de los estudiantes a través de la preparación de prácticas operativas y discursivas que ellos realizarán. Este proceso debe ser planificado a partir del contexto del aula, de los conocimientos previos, el uso de medios y las motivaciones que tenga el estudiante. La autonomía del profesor en su quehacer en el aula es su responsabilidad, pues es quien visibiliza los conflictos de los estudiantes para poner en funcionamiento indicadores que permiten controlar y mejorar las propuestas didácticas (Godino, 2011).

En las instituciones educativas generalmente se percibe un ambiente de negligencia y desmotivación entre los estudiantes, posiblemente por lo aburridas que les parecen las clases, o la falta de interés tanto del maestro como del estudiante en los procesos de enseñanza y aprendizaje de las matemáticas. Ante esa situación, se hace imprescindible la implementación de diversas y novedosas estrategias por parte del docente, que conlleven a un incremento de los aprendizajes y el mejoramiento en la calidad de la educación. Todos estos factores despertaron el interés deinvestigar acerca de estos procesos, cuyo objetivo central consiste en establecer las características de las prácticas de enseñanza y aprendizaje desde la teoría de las situaciones didácticas en la resolución de problemas de factorización para el grado octavo. Específicamente esta investigación responde a la pregunta: ¿Cuáles son las características de las prácticas matemáticas que se requieren en la resolución de problemas de factorización para el grado octavo?

La resolución de problemas, a pesar de ser recomendada por todas las instancias que tienen que ver con el currículo, como NCTM (2014), MEN (Lineamientos curriculares, 1998), ha 
sido muy poco implementada en la escuela. Muchos autores han aportado la ruta a seguir. Blanco (1996) menciona que se destacan: Dewey, con el modelo de resolución de problemas; Wallas, que formula cuatro fases de resolución de problemas; y Polya, que propuso las cuatro fases para la resolución de problemas. Por su parte, Schoenfeld, a diferencia de Polya, lo concibe como un proceso no lineal basado en estrategias heurísticas (Blanco, 1996).

Los productos notables son la introducción a la factorización, y tienen una connotación de dificultad en los estudiantesdeoctavogradodelaInstitución Educativa Técnica Antonio Nariño (IETAN), de Moniquirá, posiblemente por la forma como el maestro involucra al estudiante en este conocimiento, y por el uso o no de las diferentes representaciones en esta temática. Es imperativo encontrar estrategias, así como la mediación significativa de diferentes representaciones: la algebraica, la geométrica y la analítica; debido a que su carácter visual y su facilidad perceptiva permiten revelar regularidades que sin ellas sería difícil comprender. La elaboración y manipulación de materiales que muestran la representación geométrica de los productos notables, facilitan el conocimiento al estudiante. La relación entre el pensamiento geométrico-métrico y variacional al representar cada producto notable, puede permitir la conexión con la solución cuadrática de los mismos.

La investigación sigue un enfoque mixto, combina técnicas cuantitativas y cualitativas; parte del estudio es naturalista, que permite comprender cómo se aprenden estas nociones en un caso particular; dicho enfoque está basado en la teoría fundamentada de Strausss y Corbin (2002). La población con la que se desarrolló la investigación, fueron estudiantes de grado octavo y profesores del área de la Institución Educativa Técnica Antonio Nariño de Moniquirá (Boyacá). Los instrumentos de recolección de la información fueron: un cuestionario, talleres de aula a partir de la teoría de las situaciones didácticas, observación participante, registros narrativos de los investigadores y registros mecánicos a través de fotografías, audio y video. Se hizo también un análisis de textos de matemáticas de Educación Básica.

\section{Fundamentación Teórica. Estado del Arte}

Entre los antecedentes, se encuentra Fernández \& Mejía (2010), quienes realizaron una investigación en la que analizan los textos escolares para considerar los discursos matemáticos y las propuestas de enseñanza alrededor de la factorización de polinomios y las cónicas. Allí se plantea el problema de la escasa integración de las TIC en las temáticas de los textos escolares, no solo en nuestro país, pues es una problemática generalizada.

Robles (2015) realizó una investigación para caracterizar las prácticas evaluativas de los docentes del área de matemáticas de una institución educativa, tomando como referente las pruebas SABER. Al caracterizar las prácticas de los docentes de esa institución, analizó las pruebas internas aplicadas en el año 2013 y comparó sus contenidos con las pruebas SABER elaboradas por el ICFES,
Los productos notables son la introducción a la factorización, y tienen una connotación de dificultad en los estudiantes de octavo grado de la Institución Educativa Técnica Antonio Nariño (IETAN), de Moniquirá 


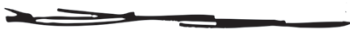

Ramos (2014) realizó una investigación a través de la reflexión docente sobre la enseñanza del álgebra, en un curso de formación continua. teniendo en cuenta tres categorías: conocimientos básicos, procesos generales y contextos. Encontró que en la Prueba de Competencias hay una debilidad generalizada en la competencia específica (Modelación, Planteamiento y Resolución de Problemas). Así mismo, en la entrevista a profundidad, los docentes manifestaron que los estudiantes tenían dificultades en la resolución de problemas, debido a que desde temprana edad no se les asignan actividades que involucren la práctica de esta competencia.

Huitrado \& Climent (2013) realizaron una investigación sobre el conocimiento profesional del profesor ante errores relativos al álgebra. Muestran resultados parciales sobre el conocimiento profesional de los profesores evaluadores puestos en acción al analizar los errores relativos al álgebra. Realizaron dos pruebas de interpretación a partir de un análisis inspirado en la teoría emergente de los datos, obtuvieron dimensiones para la caracterización de saberes en la comprensión sobre los errores. El estudio se adelantó con trescientos veintinueve alumnos $y$ cuatro profesores evaluadores de las olimpiadas de matemáticas en Zacatecas. El tratamiento de la información, recogida a través de una entrevista semiestructurada, las pruebas realizadas y las grabaciones en audio se realizaron siguiendo un proceso inductivo inspirado en la teoría emergente de los datos. Fue posible construir una serie de dimensiones sobre la interpretación del error. En los resultados incluyeron la descripción de los saberes de un profesor, estrechamente relacionados con la práctica y con el conocimiento sobre el aprendizaje de los alumnos; esta la efectuaron a través de dimensiones sobre actitudes, concepciones y posturas del profesor ante el error de los alumnos y de los saberes en la interpretación del error.

Ramos (2014) realizó una investigación a través de la reflexión docente sobre la enseñanza del álgebra, en un curso de formación continua. El objetivo principal fue estudiar la reflexión de profesores de matemáticas dentro de un curso de formación en donde la reflexión del profesor fuera efectiva. La metodología se siguió dentro del paradigma cualitativo, centrado en el análisis de contenido; donde se estudió la reflexión considerando dimensiones como las tareas matemáticas escolares y el conocimiento didáctico del docente. El curso formativo se realizó con participación de siete docentes de niveles educativos primaria, secundaria y superior. Para el análisis, seleccionaron una pareja de docentes que imparte clases en primaria y secundaria. Se utilizaron diversas fuentes de datos como: portafolios y diarios de aprendizaje de los docentes, grabaciones de las sesiones del curso, cuestionarios, entre otros. Uno de los resultados del estudio fue la caracterización de profesor reflexivo, considerando los aportes de diversos investigadores dedicados al área. Un resultado fue considerar una variedad de indicadores en un curso formativo que pretende fomentar la reflexión. Finalmente, observaron que las docentes perciben la importancia de profundizar en su conocimiento matemático para la enseñanza, con el fin de afrontar situaciones conflictivas 
de su práctica, lo que las hace mirar el álgebra de manera más funcional.

En los resultados de esas investigaciones, se aprecia que las actividades que proponen los textos, enfatizan los ambientes de lápiz y papel, y no incluyen el uso de TIC; y en ellas se afirma que los estudiantes tienen debilidades y que no solucionan problemas eficazmente.

\section{Referentes teóricos}

El significado etimológico de la palabra enseñanza, según el Diccionario de Uso del Español (Moliner 2007), expresa "del latín insignare, señalar. Hacer que alguien aprenda cierta cosa: comunicar a alguien sabiduría, experiencia, habilidad para hacer algo, hábitos, etc." (p. 1183). Rogers (citado en Diez, 1995) conceptúa que la enseñanza "constituye una actividad relativamente importante y considerablemente encarecida. El objetivo de la enseñanza no puede ser otro que el de facilitar el cambio y el aprendizaje” (p. 74). El docente también desarrolla un aprendizaje, dado que tiene la oportunidad de explorar los conocimientos previos y confrontarlos con los adquiridos, avanzar hacia un nuevo aprendizaje partiendo de la experiencia y el contacto con los estudiantes, lo cual le permite transformar la praxis y el saber. En palabras de Freire (citado en Jiménez, 2010), "Quien enseña aprende al enseñar y quien aprende enseña al aprender” (p. 72). Al respecto, Jiménez (2010) afirma que

[...] debe haber una constante retroalimentación de la práctica educativa de tal manera que se comprenda que siempre hay algo por mejorar y por aprender en la cotidianidad de la escuela y que es la comprensión de la práctica la que genera información que permite revaluar algunas acciones repetitivas y pasivas, así como viejos mitos (p. 72).

El autor advierte que este cambio depende en gran medida de la actitud proactiva del docente, que se manifiesta a través del compromiso de mejorar, estar dispuesto a autocriticarse, reconocer sus propios errores y superarlos mediante la transformación de su práctica pedagógica.

Desde otra perspectiva, los cognitivistas consideran que el aprendizaje puede no tener manifestación externa; para ellos, el aprendizaje es considerado como la alteración de las estructuras mentales de los esquemas conceptuales. Desde los enfoques conductuales, el aprendizaje es considerado como un cambio de conducta. A partir de estas tendencias, el aprendizaje matemático se consideraría que es cambiar conductas; se reitera el uso de tareas de cálculo partiendo de destrezas simples hasta conseguir destrezas más complejas. De otro lado, las interpretaciones cognitivas contraponen esta tendencia al considerar que aprender matemáticas consiste en alterar las estructuras mentales; además, dan importancia al aprendizaje de conceptos (Flores, s.f.).

Gagne (citado en Gutiérrez, 1989) expresa que "el aprendizaje consiste en un cambio en la disposición o capacidad humana, con carácter de relativa permanencia y que no es
El significado etimológico de la palabra enseñanza, según el Diccionario de Uso del Español (Moliner 2007), expresa "del latín insignare, señalar. Hacer que alguien aprenda cierta cosa: comunicar a alguien sabiduría, experiencia, habilidad para hacer algo, hábitos, etc." 
atribuible simplemente al proceso de desarrollo". El interés fundamental, además del aprendizaje, debe ser determinar las condiciones que lo encierran. En el proceso de enseñanza, se deben garantizar los recursos mínimos para que haya un verdadero aprendizaje de los estudiantes, y, a su vez, puedan madurar en sus procesos mentales; y por otra parte, el docente, a través de la autorreflexión, descubre la oportunidad de (re)significar su práctica pedagógica (Jiménez, 2002).

Díaz y Hernández (1999) sugieren que

Las principales estrategias de enseñanza que el docente puede emplear con la intención de facilitar el aprendizaje significativo de los alumnos son: [fijar] objetivos o propósitos del aprendizaje, [elaborar] resúmenes, ilustraciones, organizadores previos, preguntas intercaladas, pistas topográficas y discursivas, analogías, mapas conceptuales, redes semánticas y uso de estructuras textuales (p. 3).

Uno de los factores influyentes en la enseñanza es la investigación en el aula, partiendo de la reflexión del maestro desde su quehacer y con el compromiso de considerar factores que afectan tanto a la enseñanza como al aprendizaje, a través de una mirada cercana a los problemas que allí se presentan. La interacción con los estudiantes y el diálogo entre colegas, es lo que [...] "permite la (re)significación y la construcción de nuevos saberes sobre la práctica pedagógica del profesor de Matemática de Educación Básica y Media” (Jiménez, 2005, p. 147). Al respecto, Mora (citado en Urbano,
2011) sostiene que "las prácticas pedagógicas deben ser repensadas en presencia de la realidad cambiante, pues la escuela, el docente y el saber pedagógico sufren una de las crisis más significativas" (p. 1).

Entre los recursos y materiales que utiliza el docente en su práctica se encuentra el libro de texto; es un recurso al que los maestros le consideran de gran valor y utilidad. Campanario (2001) afirma que "para muchos profesores la elección de un libro de texto supone su decisión curricular más importante, por lo que no es raro que este instrumento ejerza un efecto poderoso sobre sus enfoques docentes y sobre las estrategias de aprendizaje de los alumnos" (p. 352). Esta elección se encuentra enmarcada en varios factores tales como gustos del discente o la disponibilidad de biblioteca de la institución; generalmente esta última, dado que, en el caso de la institución aquí mencionada, no cuenta con colecciones actualizadas de textos escolares. De igual forma, "los libros de texto escolares constituyen la fuente inmediata donde se acumula la experiencia práctica de los profesores $y$, en cierta medida, los resultados de la investigación" (Godino, Font y Wilhelmi, 2006, p. 132).

Los libros de texto ofrecen opciones de actividades en el aula y permiten su diseño, a través de distintos materiales, guías de trabajo, y el uso de software aplicativos para el trabajo en la resolución de problemas en matemáticas; pero claro, esto depende mucho de la creatividad del profesor. Polya (citado en Alfaro, 2006 sostiene 
que en este ejercicio matemático muchos docentes no encuentran progreso en el estudiante, puesto que es probable que este no tenga deseos de resolverlo; sin embargo, un método apropiado es que el profesor sea modelo para la resolución de problemas, a través del cuestionamiento a partir de preguntas durante su resolución; la elección de problemas adecuados y con el nivel de dificultad apropiado para el estudiante.

Godino, Batanero \& Font (2009) proponen la Teoría de las Funciones Semióticas, también llamado Enfoque Ontosemiótico (EOS). "El punto de partida del EOS es la formulación de una ontología de objetos matemáticos que tiene en cuenta el triple aspecto de la matemática como actividad de resolución de problemas, socialmente compartida, como lenguaje simbólico y sistema conceptual lógicamente organizado"(p. 4). El conocimiento común que se pretende enseñar a los estudiantes, hace parte de los conocimientos didáctico-matemáticos que debe tener un profesor. Del mismo modo, estos autores formulan los seis indicadores de idoneidad que deben ser aplicados para el análisis y desarrollo de una unidad didáctica; en su orden, son: idoneidad epistémica, cognitiva, interaccional, mediacional, emocional y ecológica (Godino, 2011).

Teniendo como referente dicha teoría, se hizo una caracterización de los libros de texto usados en la Institución y que hacen parte del significado institucional local por medio de la idoneidad epistémica donde emergen los distintos tipos de objetos matemáticos primarios (elementos lingüísticos, situaciones problemas, proposiciones, procedimientos y argumentos). De igual forma, el análisis permite caracterizar la faceta institucional de los conocimientos relacionados con la temática concerniente al grado octavo. En una primera fase, se empleó la configuración epistémica y cognitiva del EOS, que permite visibilizar los objetos matemáticos que intervienen en las prácticas, para conocer la configuración epistémica pretendida por los docentes de la Institución.

\section{Resultados preliminares}

Los hallazgos de esta investigación dan cuenta de la relevancia que dan los maestros a la explicación ordenada y sistemática de los contenidos de los libros de texto, en la enseñanza del álgebra y por eso los siguen.

\section{Análisis de los textos escolares}

Los textos analizados son los que están usando los docentes en el grado octavo en la IETAN de Moniquirá; sirven de fundamento para reconocer los objetos matemáticos primarios en los libros, desde la configuración epistémica de este enfoque; además, la forma en que se presentan estos contenidos y algunos aspectos que están ausentes en estos. El análisis realizado presenta únicamente, los niveles uno y dos que corresponden a sistemas de prácticas, tomando como elemento central la actividad de resolución de problemas y la configuración de objetos y procesos matemáticos, emergentes e intervinientes en las prácticas matemáticas.
Godino, Batanero \& Font (2009) proponen la Teoría de las Funciones Semióticas, también llamado Enfoque Ontosemiótico (EOS). 
En los libros de texto 1V09, 2S03 y 3C86, de contenidos de álgebra, se destacan las propiedades, procedimientos, definiciones formales y el uso de la representación geométrica de expresiones algebraicas.

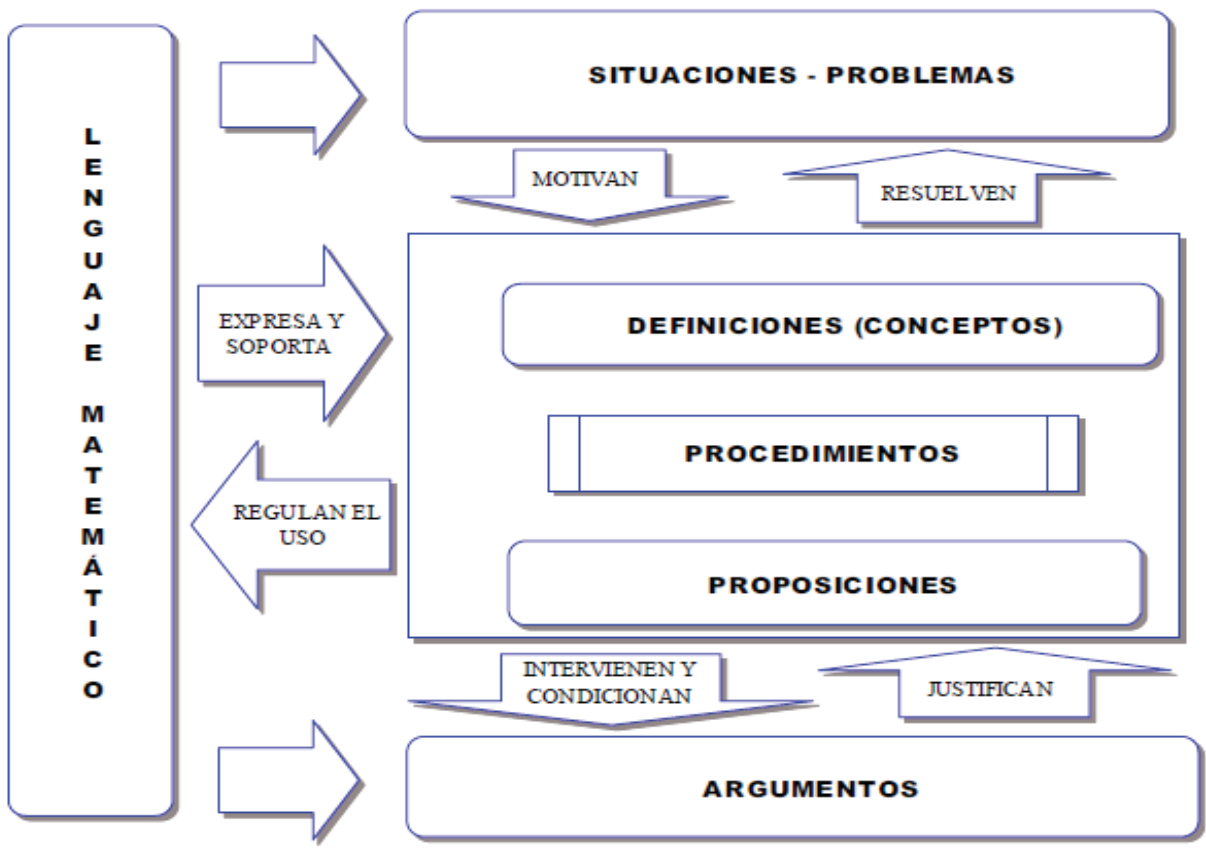

Figura 1. Configuración epistémica. Fuente: Godino, J. Batanero, C y Font, V. 2009 p. 7

Este análisis se realiza teniendo en cuenta la configuración epistémica (ver figura 1) constituido por situacionesproblemas, lenguajes, conceptos, proposiciones, procedimientos $\mathrm{y}$ argumentos.

Los libros de texto analizados en la investigación corresponden a: álgebra de Ortiz (2009), álgebra de Bautista (2003) y álgebra de Baldor (1986). Los códigos (1V09), (2S03) y (3C86) corresponden a cada texto en su orden respectivo y se usan para simplificar la lectura. Para los resultados del análisis de los textos, se hizo la descripción de los contenidos y actividades de los productos notables y, posteriormente, el análisis de los tipos de representación utilizados. Inicialmente se mencionan los elementos del contenido de los textos relacionados con los productos notables y la representación geométrica y algebraica, de acuerdo con las categorías propuestas por la Teoría de las Funciones Semióticas (Font \& Wilhelmi, 2007). Del mismo modo, se resume el desarrollo de los aspectos teóricos de los productos notables en los libros; se presenta un análisis unificado de los contenidos.

Enloslibros de texto 1V09,2S03y3C86, de contenidos de álgebra, se destacan las propiedades, procedimientos, definiciones formales y el uso de la representación geométrica de expresiones algebraicas. En el análisis de estos libros de texto, se destaca un solo elemento de geometría para cada producto notable, y se relaciona con conceptos algebraicos consistentes. Sin embargo, en el texto 3C86 la representación geométrica solamente aparece para dos productos notables. Se designa cada producto notable 
en forma algebraica con letras minúsculas; así mismo, se dan ejemplos procedimentales para cada uno.

La representación semiótica de los productos notables en los tres (3) textos es de dos tipos, algebraico y geométrico. En la representación algebraica hay expresiones algebraicas que pueden representar el lado de un cuadrado, de un rectángulo o un cubo; o las áreas de los primeros, o el volumen del cubo. Para la representación geométrica usan cuadrados, rectángulos y cubos. Explicitan por medio de dichas representaciones la descomposición de los lados y las áreas de las mencionadas figuras.

En los textos 1V09 y 2S03, se presentan los contenidos relativos a los cuatro productos notables en mención, pero hay un vacío de contenido frente a la ilustración del cuadrado de la diferencia de un binomio, pues no aparece la representación geométrica.

En las propiedades y procedimientos descritos en los contenidos, se destacan en 1V09 y 2S03 las representaciones algebraica y geométrica de los productos notables a través de la descripción e ilustración de cada uno, y tiene un componente visual desde las mismas representaciones. En 3C86, solo aparecen las representaciones geométricas de dos productos notables.

En lo referente al lenguaje y representaciones, se observa que los tres (3) textos tienen un lenguaje algebraico y geométrico sencillo; además, las dos representaciones de los productos notables se observan en varios pasos. En 1V09, se muestra paso a paso, tanto el procedimiento algebraico, como el geométrico, a través de la descomposición de las figuras para cada producto notable. En 2S03, presentan la figura dividida en colores y una breve explicación de los sectores de la misma. En 3C86, visualizan dos productos notables geométricamente y señalan los pasos, desde el dibujo de cada figura.

Este análisis destaca, en el contenido, los elementos de álgebra y geometría que se refieren especialmente a aspectos conceptuales relacionados con los productos notables.

Sobresale ampliamente el álgebra de Ortiz porque se ha preocupado por incluir una sección de tecnología, que permite ver la exploración de una de las aplicaciones más comunes de los polinomios, como es la transmisión de datos. Además, presenta el resumen, refuerzo y la evaluación del tipo prueba Saber, Pisa y Timss, completamente aplicada a la geometría. Así mismo, el texto 2S03 se destaca por la inclusión de las aplicaciones geométricas de perímetros, áreas y volúmenes. La editorial cultural venezolana destaca la vasta ejercitación de procedimientos algebraicos.

\section{Análisis de los tipos de representaciones utilizadas}

Las representaciones de los objetos bidimensionales en los libros de texto, permiten que los estudiantes vinculen los conceptos algebraicos y geométricos correspondientes; se esperaría que los estudiantes identifiquen las representaciones de los productos notables y los asocien con lo geométrico
La representación semiótica de los productos notables en los tres (3) textos es de dos tipos, algebraico y geométrico. 
La investigación en curso, titulada "prácticas matemáticas en la resolución de problemas sobre factorización" se apoyó en el cuestionario aplicado a 191 estudiantes del grado octavo, que buscaba conocer sobre las prácticas de enseñanza y aprendizaje en la resolución de problemas de factorización. que se quiere mostrar. En los libros analizados prevalece la representación única (algebraica). Se observa en los tres (3) textos que las actividades son abundantes en cuanto a la cantidad de ejercicios propuestos, pero es evidente la inexistencia de otras representaciones gráficas o curvas en los temas referentes a los productos notables y su conexión con la factorización.

Algunas situaciones en la enseñanza de la matemáticatienden a mantenerseestáticas; y la ejercitación de procedimientos algorítmicos tampoco es la excepción. Las prácticas de enseñanza de los docentes de matemáticas, según Mejía (2012), comúnmente hacen uso de métodos de factorización como de manipulación de expresiones algebraicas con lápiz y papel. Este procedimiento tiende a desconectar la factorización de otros conceptos y, además, aleja al estudiante del uso de otras representaciones que fortalecen el aprendizaje. Por esta razón, algunos docentes incorporan en el aula el uso de las Tecnologías de la Información y la Comunicación (TIC) como posibilidades reales para dinamizar el aprendizaje de los productos notables y, en general, el álgebra elemental.

Con base en las debilidades anteriores identificadas en los libros de texto, se presenta la propuesta a través de la integración de las representaciones algebraica y geométrica enlazada con otro tipo de representaciones que permitan ampliar la visión de los productos notables: la representación cartesiana en el software Geogebra, la cual completa una terna que da cuenta de dicha mirada global, fortaleciendo simultáneamente el uso del pensamiento espacial y el algebraico variacional.

\section{Análisis del cuestionario aplicado a los estudiantes}

La investigación en curso, titulada "prácticas matemáticas en la resolución de problemas sobre factorización", se apoyó en el cuestionario aplicado a 191 estudiantes del grado octavo, que buscaba conocer sobre las prácticas de enseñanza y aprendizaje en la resolución de problemas de factorización. Contiene once (11) preguntas "cerradas" por la facilidad de codificación y análisis, acompañadas de preguntas "abiertas", las cuales profundizan la opinión de los estudiantes frente a las categorías a evaluar. El instrumento hace parte del primer objetivo de la investigación y fue analizado con el software SPSS, donde se consolidó la información que permitió identificar que existe igual número de niñas y niños $(48,7 \%)$, con edades comprendidas entre los $13(44,5 \%)$ y 14 $(28,8 \%)$ años de edad en su mayoría. Así mismo, se evidencia que un $56 \%$ han tenido dificultades con el aprendizaje del álgebra, porque no la entienden $(36,6 \%)$ o les parece confusa $(16,6 \%)$, provocadas principalmente por la indisciplina en clase $(16,2 \%)$ y la falta de interés $(10,5$ \%). Sin embargo el 80,6 \% de los chicos, considera que el aprendizaje del álgebra es importante para resolver problemas de la vida diaria. La mayoría relaciona el aprendizaje del álgebra con las compras, con las profesiones, facturación de recibos, utilidad en el futuro, otros pocos manifiestan que no saben; aproximadamente.

La cuarta parte no tiene clara la utilidad del álgebra. Estas respuestas permiten intuir que la enseñanza del álgebra por estos profesores se centra en la exposición de contenidos, donde la resolución de 
problemas en contexto no es tenida en cuenta; por eso, los estudiantes hacen afirmaciones sobre supuestas utilidades, fuera de contexto.

La gran mayoría de los estudiantes coinciden en que las clases de álgebra que reciben se fundamentan en las explicaciones de sus docentes, hacen uso de algunas representaciones geométricas, con escasa utilización de material de apoyo y, en el menor de los casos, el empleo de la calculadora; en contraste, no se utilizan las nuevas tecnologías. Igualmente, el desarrollo de la clase es magistral, pues se centra en el docente con escasa participación de los estudiantes, prevaleciendo la enseñanza tradicional. Desde la perspectiva del EOS, el "significado institucional" implementado que prevalece es el modelo pedagógico tradicional (Godino, Batanero \& Font, 2009, p. 5).

El tema más recordado por la mayoría de los estudiantes preguntados es el de las operaciones con polinomios; tal vez, por ser el más reciente al momento de la aplicación del cuestionario. Un menor porcentaje, también recuerdan temas como el teorema de Pitágoras, la factorización, el triángulo de Pascal y los productos notables.

A la mayoría de los estudiantes les agrada que el profesor les explique los temas de álgebra y, en menor porcentaje, les satisface que el profesor les permita el trabajo en grupo, el desarrollo de talleres y el manejo estricto de la disciplina para poder concentrarse, al momento de resolver problemas de matemáticas. Sin embargo, al cuestionarlos sobre aquello que les disgusta de sus clases, un buen número de ellos no responde, quizás, por el temor a posibles represalias de sus docentes. Quienes respondieron esta pregunta, consideran que les disgusta no entender el tema de clase, y que como consecuencia, se distraen y pierden el interés por el estudio. A ellos no les gustaría que su profesor los regañe en clase, que les dejen mucho trabajo y les gustaría que las clases no sean monótonas. De igual manera, expresan que se cansan de copiar y les gustaría que el profesor busque alternativas con el apoyo de las TIC. Parra (2016) afirma: que "La implementación de la didáctica de la matemática es imprescindible para el abandono de la educación tradicional que origine un cambio de actitud de docentes y estudiantes para el mejoramiento de la enseñanza y el aprendizaje de las matemáticas" (p. 27). En las respuestas dadas por los estudiantes, piden que se realicen juegos, lúdicas y más explicaciones de los temas de álgebra. Ellos solicitan actividades donde se incorpore la lúdica en el aula con cierto nivel de exigencia y disciplina, y el desarrollo de actividades en el aula para evitar tareas en casa.

En cuanto al aprendizaje, la mayoría de los estudiantes manifiesta tener dificultades en el aprendizaje del álgebra. Pero no tienen claro cuáles dificultades presentan, posiblemente por olvido de las temáticas. Aproximadamente, la tercera parte del grupo manifiesta que no entiende las temáticas que les presenta el docente; unos cuantos afirman que no prestan atención a la clase; otros dicen que tienen dificultades con la simbología algebraica. Uno de los indicadores de idoneidad cognitiva del EOS, son los conocimientos
La gran mayoría de los estudiantes coinciden en que las clases de álgebra que reciben se fundamentan en las explicaciones de sus docentes, hacen uso de algunas representaciones geométricas, con escasa utilización de material de apoyo y, en el menor de los casos, el empleo de la calculadora; en contraste, no se utilizan las nuevas tecnologías. 
Uno de los componentes de idoneidad cognitiva del EOS, es el referente a los contenidos pretendidos por el maestro; es decir, las temáticas que se trabajan con los estudiantes. previos; existe la posibilidad de que los estudiantes no tengan los conocimientos previos necesarios para el estudio del álgebra, pero el maestro no hace una indagación anterior, o no lo planifica en el momento en que se inician las clases, pues así lo manifiestan los estudiantes.

Uno de los componentes de idoneidad cognitiva del EOS, es el referente a los contenidos pretendidos por el maestro; es decir, las temáticas que se trabajan con los estudiantes. Aquí tienen una dificultad alta, pues lo mencionan los estudiantes en el cuestionario; por tanto, es importante que el maestro redireccione el proceso de enseñanza. Al respecto, Godino (2011) afirma que

El logro de una idoneidad alta en una de las dimensiones, por ejemplo, la epistémica, puede requerir unas capacidades cognitivas que no posean los estudiantes a los que se dirige la enseñanza. Una vez logrado un cierto equilibrio entre las dimensiones epistémica y cognitiva es necesario que la trayectoria didáctica optimice la identificación y solución de conflictos semióticos (p. 7).

Las facetas que posibilita el EOS no son factores aislados o independientes, puesto que hay una estrecha relación entre ellas. Entre los componentes e indicadores de idoneidad están las facetas epistémica-cognitiva-afectiva las cuales refieren la importancia de reflexión de los estudiantes sobre sus razonamientos en el proceso de resolución de problemas, hasta ser capaces de aplicar las estrategias empleadas en otros problemas y contextos (Godino, 2011).
La mayoría de los estudiantes manifiesta no conocer ambientes para aprender álgebra en internet. Además, no contesta a la pregunta cuáles ambientes conoce; aproximadamente la cuarta parte menciona los tutoriales, y un pequeño porcentaje mencionan la calculadora. Así mismo, la mayoría no contesta a la pregunta de qué forma aprende conceptos algebraicos con esos ambientes. El significado institucional implementado por el docente durante las clases, en cuanto al uso de recursos tecnológicos, indica que la escasa implementación no permite determinar el abordaje de ciertos problemas y la configuración de objetos y procesos, lo cual implica nuevas formas de representación, argumentación y generalización. El uso de un recurso tecnológico implica afectar positivamente la forma como interactúa el docente y el estudiante, su interés y motivación en el aprendizaje (Godino, 2011).

\section{Algunas conclusiones}

En los libros de texto de matemáticas analizados, aparecen las representaciones de los productos notables, manteniendo un aspecto global de los objetos, especialmente en lo algebraico y geométrico, como lo menciona Parzysz (1988, 1991); la representación algebraica y geométrica de los productos notables permite al estudiante reconocer a primera vista el objeto en cuestión; pero, al mismo tiempo, presenta un aspecto negativo, ya que los estudiantes se enfrentan a representaciones únicas y en una misma posición; ellos presentan dudas sobre estas representaciones, no tienen idea del porqué las expresiones algebraicas están representadas así, y es evidente que no la representarán de otra forma. 
En los libros analizados, las actividades muestran representaciones únicas y no describen características para todos los productos notables. Las representaciones gráficas de los productos notables en otros contextos no se tienen en cuenta. Lo anterior muestra que deben buscarse opciones que amplíen esta visión en la enseñanza del álgebra; en palabras de Parzysz (1988) "[...] no quedarse esclavos de dibujos estereotipados, que han perdido una gran parte de su poder operacional” p. 90. Dicha posibilidad tendría que enmarcarse en las distintas representaciones matemáticas posibles y en las propiedades analíticas que surgen a partir de ellas.

Como se ve, el significado institucional, desde el papel ostensivo de la representación y comunicación en la geometría euclidiana, no está lo suficientemente valorado en los libros de texto; los objetos matemáticos y sus representaciones son muy elementales. Las representaciones de los productos notables tienen ciertas regularidades que no se reflejan totalmente al representarlos de formas únicas.

Las actividades propuestas en los libros de texto cubren parcialmente aspectos como actividades de razonamiento y ejercitación de algoritmos.

La posibilidad de proponer el uso de otras representaciones conectadas con la factorización, permite enriquecer la forma de abordar las temáticas e ir más allá de lo presentado en los libros, pues los tres contextos planteados permiten la exploración algebraica, geométrica y cartesiana de los productos notables; a su vez, amplían la visión de los estudiantes frente a la resolución de problemas. Duval (1999) conceptúa que se incrementa la comprensión de los sujetos cuando se diversifican las representaciones semióticas de un mismo objeto. Las diversas representaciones de los productos notables, en cualquier contexto, permiten la exploración en busca de propiedades inherentes, que serían escasas con una o dos representaciones. Cada uno de estos sistemas, tiene potencialidades y limitaciones, por lo que su utilización conjunta es esencial para producir diferentes sentidos o bien para escoger uno de ellos.

El análisis de los libros de texto se constituye en un material interesante en el estudio del significado institucional que el maestro pretende ofrecer a los estudiantes, frente a las temáticas establecidas en el currículo. De igual forma, el componente de idoneidad cognitivo del EOS, advierte sobre la importancia que debe dar el maestro a los conocimientos previos que tienen los estudiantes al inicio del año escolar; esto se convertirá en un componente fuerte para el maestro en sus observaciones y en la investigación in situ.

En la enseñanza, el uso de recursos tecnológicos permite, desde la faceta epistémica, cognitiva y afectiva, un acercamiento al conocimiento que adquiere el estudiante, una reflexión sobre su aprendizaje y un mejoramiento en el ambiente de las clases.

Al comparar los significados pretendidos con los implementados, se observa que no se encuentran en la zona de desarrollo potencial de los estudiantes, dado que la dinámica de la
Como se ve, el significado institucional, desde el papel ostensivo de la representación y comunicación en la geometría euclidiana, no está lo suficientemente valorado en los libros de texto; los objetos matemáticos y sus representaciones son muy elementales. 
Se diseñaron tres talleres, a partir del análisis de los tres textos escolares y el cuestionario realizado a 191 estudiantes de octavo grado, con el objetivo de crear una teoría que permita explicar las situaciones del aula de clase frente a los productos notables clase se centra en el docente y la faceta personal e institucional se encuentra desarticulada de la dialéctica docente discente con un frágil espacio de diálogo, confrontación y socialización.

\section{Prospección educativa}

Del análisis anterior, se percibe que el docente debe brindar el conocimiento al estudiante de tal forma que reconozca la globalidad de este y la articulación de distintas representaciones; Duval (citado en Font, 2009) menciona que la diversificación de representaciones semióticas de un mismo objeto aumenta la comprensión de los sujetos, además destaca la existencia de diversos sistemas de representación ligados a un mismo objeto matemático. Cada uno de estos sistemas tiene potencialidades y limitaciones, por lo que su utilización conjunta es esencial para producir diferentes sentidos o bien para escoger uno de ellos.

El uso de tecnología de la App Geogebra permite la posibilidad de análisis en otros ambientes dinámicos; además, el uso delas diferentes representaciones en la segunda fase dará la posibilidad de exploración y conceptualización de los productos notables. Este proceso procura ser cíclico y continuamente retroalimentado a través de la reflexión en la práctica educativa por medio de la renovación por parte de los investigadores y expertos, siendo considerada conveniente en la enseñanza y aprendizaje de los productos notables. Este material fue usado en la investigación aquí descrita, lo cual cambió en mucho la situación.

En este sentido, el uso de esta herramienta permitió proporcionar el medio didáctico en donde el estudiante construye su conocimiento, con apoyo en la teoría de las situaciones didácticas de Brousseau en la cual plantea una forma de "modelar" el proceso de enseñanza y aprendizaje, en donde el docente y el estudiante definen unas reglas y acciones implícitas y se establece la interrelación profesor-estudiante-medio didáctico.

Se diseñaron tres talleres, a partir del análisis de los tres textos escolares y el cuestionario realizado a 191 estudiantes de octavo grado, con el objetivo de crear una teoría que permita explicar las situaciones del aula de clase frente a los productos notables; también, se pretendió la identificación de las diferentes representaciones: geométrica, algebraica y gráfica. Se reconocieron algunas regularidades presentes en cada una de las diferentes representaciones. Estas representaciones: numérica, algebraica $\mathrm{y}$ en el plano cartesiano, permiten al estudiante un mejor acercamiento frente al aprendizaje y al problema de la diversidad de representaciones semióticas en matemáticas. Al respecto, D' Amore (2006) afirma que "Sin duda, el uso de distintas representaciones y su progresiva articulación enriquecen el significado, el conocimiento, la comprensión del objeto, pero también su complejidad" (p. 13). Como se mencionó anteriormente, aquí solo se presentan resultados parciales de la investigación; pero se deja abierta la posibilidad de usar en la clase de álgebra estos tres tipos de representaciones semióticas e investigar más en profundidad los resultados y cambios en el aprendizaje y la actitud de los estudiantes hacia el álgebra. 


\section{Referencias}

Alfaro, C. (2006). Las ideas de Polya en la resolución de problemas. Cuadernos de investigación en educación matemática, 1(1)1-13.

Baldor, A. (1986). Álgebra. Venezuela: Cultural venezolana

Bautista, M. Salgado, D. Nivia, L. Acosta, M. \& Orjuela, J. (2003). Álgebray Geometría I. Bogotá: Santillana.

Blanco, J. (1996). La resolución de problemas. Una revisión teórica. Revista Summa, 21, 12-13.

Campanario, J. M. (2001). ¿Qué puede hacer un profesor como tú o un alumno como el tuyo con un libro de texto como éste? Una relación de actividades poco convencionales. Enseñanza de las Ciencias, 19(3), 351-364.

D' Amore, B. (2006). Objetos, significados, representaciones semióticas y sentido. In L. Radford, \& B. D'Amore (eds.), Semiotics, Culture and Mathematical Thinking. Numero special della rivista (pp. 177-196). México: Cinvestav.

DíAz, F., \& Hernández, G. (1999). Estrategias docentes para un aprendizaje significativo. México: McGraw-Hill. Recuperado de http://www.urosario.edu.co/CGTIC/ Documentos/estategias_docentes.pdf

DiEz, J. (1995). Hacia un modelo comprensivo de prácticas de enseñanza en la formación inicial del maestro. (Tesis Doctoral). Universidad Complutense, Madrid, España.

Duval, R. (1999). Semiosis y Pensamiento Humano. Registros semióticos y aprendizajes intelectuales. Cali: Universidad del Valle.

Fernández, E., \& Mejía, M. F. (2010). Análisis de textos escolares para el diseño de situaciones de enseñanza. Encuentro colombiano de Matemática Educativa. Bogotá, Colombia.

Font, V., Godino, J., \& D’Amore, B. (2007). Enfoque ontosemiótico de las representaciones en educación matemática. Recuperado de http://www.ugr.es/ jgodino/funcionessemioticas/enfoque_ontosemiotico_representaciones.pdf

FonT, V. (2009). Algunos puntos de vista sobre las representaciones en didáctica de las matemáticas. Recuperado de http://www.cimm.ucr.ac.cr/ojs/index.php/eudoxus/article/view

Florez, P. (s.f.). Aprendiraje en Matemáticas. Recuperado de http://www.ugr.es/ pflores/ textos/cLASES/CAP/APRENDI.pdf

Godino, J. D., Font, V. \& Wilhelmi, M. R. (2006). Análisis ontosemiótico de una lección sobre la suma y la resta. Revista Latinoamericana de Investigación en Matemática Educativa, 9 (Especial), 132-156.

Godino, J. D., Font, V. \& Wilhelmi, M. R. (2007). Análisis didáctico de estudio matemático basado en el enfoque ontosemiótico. Publicación (en prensa). Brasil: Ulbra.

Godino, J., Batanero, C., \& Font, V., (2009). Un enfoque ontosemiótico del conocimiento y la instrucción matemática. The international Journal on Mathematics Education, 39, 127-135. Recuperado de http://www.ugr.es/local/jgodino

Godino J. (2011). Indicadores de idoneidad didáctica de procesos de enseñanza y aprendizaje de las matemáticas. XIII Conferência Interamericana de Educação Matemática (CLAEM-LACME), Recife, Brasil.

GutiérreZ, R. (1989). Psicología y aprendizaje de las ciencias. El modelo de Gagné. Enseñanza de las Ciencias, 7(2), 147-157. 
Hernández R., Fernández C., \& Baptista P. (2010). Metodología de la Investigación (5ª ed.). México: McGraw-Hill.

HutrRado \& Climent (2013). Conocimiento del profesor en la interpretación de errores de los alumnos en álgebra. $P N A, 8(2), 75-86$.

JimÉnEZ, A. (2002). Quando professores de Matemática da escola e da universidade se encontram. Tese de doutorado. Universidade Estadual de Campinas (UNICAMP); Campinas - São Paulo, Brasil.

JimÉnEZ, A. (2005). Formación de profesores de matemática: aprendizajes recíprocos escuelauniversidad. Tunja: Búhos Editores.

JimÉNEZ, A., \& HERNÁNDEZ C. (2010). Investigar para transformar la práctica: una caracterización de estilos de enseñanza en matemáticas. Cuadernos de Psicopedagogía (7), 67-80.

Moliner, M. (2007). Diccionario de uso del español (3ª ed). Madrid: Gredos.

Mejía, M. (2012). ¿Cómo se podría enseñar la factorización de polinomios integrando calculadoras simbólicas y lápiz/papel? Memorias del $13^{\circ}$ Encuentro Colombiano de Matemática Educativa. Medellín. Recuperado en http://funes.uniandes.edu. $\mathrm{co} / 2335 /$

MEN: (1998). Lineamientos curriculares. Colombia.

NCTM. (2014). Principios para la acción. Resumen ejecutivo, Mathus, C.

NiETO, J. (2002). Hacia un modelo comprensivo de prácticas de enseñanza en la formación inicial del maestro. (Tesis Doctoral). Universidad Complutense de Madrid, Madrid, España.

Ortiz, M., DíAz, S., López, J., \& LEÓn, J. (2009). Fórmula Álgebra y Geometría (1a. ed.). Colombia: Voluntad.

PARRA, L. (2016). El aprendizaje de los elementos notables de un triángulo explorando sus sistemas de representación (Tesis de maestría). Universidad Santo Tomás, Tunja, Colombia.

Parzysz, B. (1988). "Knowing" vs "seeing". Problem of the plane representation of space geometry figures. Educational Studies in Mathematics, 19, 79-92.

Ramos, E. (2014). Reflexión docente sobre la enseñanza del álgebra, en un curso de formación contínua. Recuperado de https://dialnet.unirioja.es/servlet/tesis?codigo=57549

Robles, J. (2015) Caracterización de las Prácticas Evaluativas de los Docentes del Área de Matemáticas de la fase IV de la Institución Educativa San Mateo tomando Como referente las Pruebas Saber. (Tesis de Maestría). Universidad Santo Tomás, Tunja, Colombia.

SANCHO, J., \& MartíneZ, S. (2014). La importancia de las relaciones investigadorinvestigado: El caso de las narrativas de vida profesional. Tendencias pedagógicas, (24), 225-240.

Strauss, A. \& CORBIN J. (2002). Bases de la investigación cualitativa. Técnicas y procedimientos para desarrollar la teoría fundamentada. Medellín, Colombia: Editorial Universidad de Antioquia.

Urbano, M. (2011). Experiencias Docentes Estrategia Didáctica Lúdica basada en el Computador para Enseñanza de Polinomios en Segundo Año de Educación Básica. Revista de investigación, Pensamiento Matemático, (1), 1-21. Recuperado de http://www2.caminos.upm.es/Departamentos/matematicas/revistapm/revista_ impresa/numero_1/experiencias_maria_c_urbano.pdf 